Published in final edited form as:

Curr Eye Res. 2014 June ; 39(6): 604-610. doi:10.3109/02713683.2013.859275.

\title{
Cytoprotective effect of lacritin on human corneal epithelial cells exposed to benzalkonium chloride in vitro
}

\author{
Mary M. Feng ${ }^{*}$, \\ Department of Ophthalmology, Western University, London, ON, Canada \\ Julia Baryla*, \\ Department of Ophthalmology, Western University, London, ON, Canada \\ Hong Liu, \\ Department of Ophthalmology, Western University, London, ON, Canada \\ Gordon W. Laurie, \\ Department of Cell Biology, University of Virginia, Charlottesville, VA \\ Robert L. McKown, \\ Integrated Science and Technology, James Madison University, Harrisonburg, VA \\ Negin Ashki, \\ Department of Ophthalmology, Western University, London, ON, Canada \\ Dinesh Bhayana, and \\ Department of Ophthalmology, Western University, London, ON, Canada \\ Cindy M.L. Hutnik \\ Department of Ophthalmology, Department of Pathology, Western University, Ivey Eye Institute, \\ St. Joseph's Hospital, 268 Grosvenor Street, London, ON, Canada N6A 4V2, Phone: \\ 1-519-646-6272, Fax: 1-519-646-6410 \\ Cindy M.L. Hutnik: cindy.hutnik@sjhc.london.on.ca
}

\section{Abstract}

Purpose-Benzalkonium chloride (BAK) is the most commonly found preservative in eye drops, and has been shown to cause ocular surface inflammation and toxicity. Lacritin is a human tear glycoprotein secreted from the lacrimal glands that has been found to be cytoprotective. This study was designed to determine if the presence of lacritin confers protection to a cultured human corneal epithelial (HCE) cell line, CRL-11515, and primary HCE cells after exposure to the ocular preservative agent BAK.

Materials and Methods-Recombinant human lacritin was cloned into intein fusion vectors, expressed in E. coli, and purified on chitin beads and DEAE Sepharose. Metabolic curves were

Correspondence to: Cindy M.L. Hutnik, cindy . hutnikes jhc. london. on. ca.

*M.M. Feng and J. Baryla contributed equally to this work.

Declaration of Interest

This research was sponsored by grants from the National Institutes of Health, the Glaucoma Research Society, and Pfizer. The authors have full control of all primary data and we agree to allow Current Eye Research to review this data upon request. 
established using the MTT assay after exposure of subconfluent CRL-11515 cells to BAK or lacritin. Western blot analysis of lipidated LC3 (LC3-II) provided a measure of autophagy in CRL-11515 cells exposed to lacritin and/or BAK.

Results-BAK reduced CRL-11515 cellular metabolic activity in a time and dose dependent manner. BAK-induced cellular stress was evident by elevated autophagy that increased with rising concentrations of BAK compared to control $(\mathrm{P}<0.05)$. Lacritin increased $\mathrm{HCE}$ cell proliferation at an optimal dose of $1 \mathrm{nM}$. Preconditioning HCE cells with $1 \mathrm{nM}$ lacritin for 24 hours prior to BAK exposure significantly dampened levels of LC3-II $(\mathrm{P}<0.05)$ and promoted a significant increase in cellular metabolic activity $(\mathrm{P}<0.01)$ compared to BAK alone.

Conclusions-These results suggest lacritin protects cultured HCE cells stressed with BAK. Lacritin may have the potential to be used as a topical adjunctive therapy in eyes chronically exposed to BAK.

\section{Keywords}

Lacritin; Benzalkonium chloride; Human corneal epithelial cells; Autophagy

\section{Introduction}

Many topical medications for ophthalmic use have documented surface toxicity. This often leads to significant patient morbidity, and occasionally, the discontinuation of medications despite therapeutic benefits. For chronic diseases like glaucoma, lack of compliance due to cessation of topical medical therapy can result in irreversible visual loss. Surface toxicity can be attributed, in part, to preservatives found in topical medications, most commonly benzalkonium chloride (BAK).

The reported negative clinical effects of BAK include photophobia, hyperemia, corneal edema, punctuate keratitis, gray corneal epithelial haze, pseudomembrane formation, and toxicity to corneal cells (1). BAK has been found to cause damage by several mechanisms including promoting inflammation and mediating cell death. Human corneal and conjunctival cells demonstrated significant induction of tumor necrosis factor-alpha (TNFa), an inflammatory biomarker, after exposure to low doses of BAK (2). BAK has also been reported to cause apoptosis of conjunctival (3) and corneal cells (4); at higher concentrations, cell death can also occur via necrosis $(3,4)$. BAK can remain in ocular tissues up to 48 hours after application (1) and has a cumulative effect (3). Therefore, a patient on chronic eye drops, such as a typical glaucoma patient, is more likely to experience toxicity than an individual only requiring acute and temporary therapy.

Lacritin is a secreted glycoprotein from acinar cells of the human lacrimal gland and is also expressed by conjunctival and corneal epithelial cells $(5,6)$. Being in the tear film, it has been under investigation for its protective properties on the corneal epithelium $(7,8)$. This natural tear molecule behaves as an ocular-specific prosecretory mitogen by stimulating basal lacrimation in rat lacrimal acinar cells (9). In addition, lacritin promotes human corneal epithelial (HCE) cell homeostasis (10) and stimulates HCE proliferation (8). Evidence has also been presented for selective lacritin downregulation in tears of patients 
with contact lens-related dry eye (11), blepharitis (12), and Sjögren's syndrome (13), suggesting that the presence of lacritin is integral to maintaining a healthy ocular surface.

Autophagy is a cellular process critical in maintaining homeostasis under physiological conditions. However, excessive autophagy contributes to cell injury and death $(14,15)$. It is not known whether lacritin protects human corneal epithelial cells from BAK-induced cytotoxicity. The present study was designed to determine whether lacritin protects against BAK-induced cell injury and autophagy in human corneal epithelial cells. If lacritin is found to be cytoprotective in this application, it has the potential to be developed as a natural topical adjunctive therapy in eyes exposed to chronic stressors, such as topical preservatives. This, in turn, may have a positive influence on compliance.

\section{Materials and Methods}

\section{Reagents}

All cell culture reagents were purchased from Invitrogen (Gibco BRL, Burlington, ON, Canada). LC3 antibodies and MTT were purchased from Sigma-Aldrich (Oakville, ON, Canada).

\section{Cell culture}

The human corneal epithelium (HCE) cell line CRL-11515 was purchased from the American Type Culture Collection (Manassas, VA). Primary HCE were purchased from Sciencell Research Laboratories (Carlsbad, CA). The lacritin DNA coding sequence was cloned into the IMPACTTM-CN vector pTYB1 (New England BioLabs, Inc., Ipswich, MA) to generate the lacritin-intein fusion plasmid "pLAC". Recombinant proteins were expressed in E. coli, purified on chitin beads (New England BioLabs, Inc., Ipswich, MA), and further purified on DEAE Sepharose (GE Healthcare, Little Chalfont, United Kingdom) as previously described (13). Purified proteins were freeze-dried and stored at $-80^{\circ} \mathrm{C}$ until use.

CRL-11515 cells and primary HCE cells within 5 passages from the time of purchase were cultured in a Keratinocyte-Serum Free medium. They were seeded at 105 cells onto 24 well plates and grown for 24 hours. BAK and lacritin experiments were initiated at $60 \%$ confluency as determined by the area of cell coverage under microscopy.

\section{BAK time and dose curves - cellular metabolic activity}

$60 \%$ subconfluent CRL-11515 cells were treated with $0.004 \%$ BAK for 1, 3, 10 and 30 minutes. CRL- 11515 cells were treated with $0.001 \%, 0.002 \%, 0.003 \%, 0.004 \%$, and $0.005 \%$ of BAK for 1 minute. Cellular metabolic activity was subsequently assessed with the MTT assay.

\section{Lacritin time curve - cellular metabolic activity}

$60 \%$ subconfluent CRL-11515 cells were exposed to $1 \mathrm{nM}$ lacritin for 1 minute, 15 minutes, 30 minutes, and 24 hours. Cells were then washed, and cellular metabolic activity was assessed with the MTT assay. 


\section{BAK and Lacritin - Autophagy}

$0.001 \%$ and $0.004 \%$ BAK was added to $60 \%$ subconfluent CRL-11515 cells for 1 minute and autophagy was assessed using Western blot analysis to monitor levels of lipidated LC3II. Autophagy was similarly estimated after lacritin treatment $(0.1,1,10 \mathrm{nM})$.

Three sets of experiments were performed using BAK $(0.004 \%)$ and lacritin $(1 \mathrm{nM})$. Cellular metabolic activity in CRL-11515 and primary HCE cells were measured using the MTT assay. Autophagy was assessed by Western Blotting for LC3-II.

1. BAK treatment of subconfluent HCE cells for 1 minute (BAK).

2. Simultaneous administration of $B A K$ and lacritin for 1 minute $(B A K+L)$.

3. Subconfluent HCE cells pre-incubated with lacritin for 24 hours, followed by addition of BAK for 1 minute (L24h/BAK).

\section{MTT Assay}

The MTT reduction assay was used as an index of cellular metabolic activity and was performed according to the manufacturer's instructions. Briefly, control and treatment groups were incubated in serum-free medium containing $0.4 \mathrm{mg} / \mathrm{mL}$ MTT [3-(4,5dimethylthiazol-2-yl)-2,5-diphenyltetrazolium bromide]. During this time, mitochondrial and cytosolic dehydrogenases of living cells reduced the yellow tetrazolium salt (MTT) to a purple formazan dye capable of spectrophotometric detection. After 2 to 2.5 hours, the MTT solution was aspirated and dimethylsulfoxide $(0.3 \mathrm{~mL} /$ well $)$ was added. Optical densities were read at $575 \mathrm{~nm}$ using the iMark Microplate Reader. Absorbances were normalized to the untreated control cultures, which represented $100 \%$ viability.

\section{Protein Extraction and Western Blot Analysis}

Subconfluent CRL-11515 cells treated with $0.001 \%$ or $0.004 \%$ BAK for 1 minute in the absence or presence of lacritin were lysed (50 mM Tris- $\mathrm{HCl}$ [pH 7.5], $150 \mathrm{mM} \mathrm{NaCl}, 1 \mathrm{mM}$ EDTA, $0.5 \%$ SDS, and 1:100 protease inhibitor) to extract protein. Total protein was measured by the Micro BCA Protein Assay kit (Thermo Scientific, Nepean, ON) and $20 \mu \mathrm{g}$ of protein was loaded per well. Protein was separated on a 10\% PAGE gel and transferred to a nitrocellulose membrane. Autophagy was estimated by Western blotting for the autophagy marker LC3-II using anti-LC3-II antibody (Sigma-Aldrich, St. Louis, MO) at a dilution of 1:1,000 followed by anti-rabbit horseradish peroxidase-conjugated secondary antibody $(1: 3,000)$ and signal was detected by enhanced chemiluminescence (Pierce Biotechnology, Rockford, IL) with the ChemiGenius 2 Imaging System (Syngene, Frederick, MD). To ensure equal protein loading, nitrocellulose membranes were stripped (REblot; Chemicon International, Temecula, CA) and reprobed with anti-GAPDH antibody at a dilution of 1:5,000 (Cedarlane Laboratories, Hornby, ON, Canada). Intensities were quantified using the FluorChem 8000 (Alpha Innotech, San Leandro, CA) and corrected for background. The densitometric ratio of lipidated LC3-II/GAPDH is presented. 


\section{Statistical Analysis}

All data are given as the mean $+/-$ SD of at least three experiments. Where applicable, differences between two groups were compared by the unpaired, two-tailed Student's $t$-test. For multigroup comparisons, one-way ANOVA followed by Student-Newman-Keuls test was performed. $P<0.05$ was considered statistically significant.

\section{Results}

\section{BAK-induced suppression of CRL-11515 cellular metabolic activity is time and dose dependent}

BAK promoted a time (Fig. 1A) and dose (Fig. 1B) suppression of CRL-11515 cellular metabolic activity. Higher concentrations and longer exposures of BAK enhanced suppression. CRL-11515 cells treated with $0.004 \%$ BAK for 1 minute were suppressed by approximately $40 \%$. This concentration and exposure time of BAK was utilized for the subsequent experiments.

\section{Lacritin enhanced metabolic activity of CRL-11515 cells is time dependent}

The optimal exposure time to lacritin was determined by treating CRL-11515 cells with 1 $\mathrm{nM}$ lacritin for 1 minute, 15 minutes, 30 minutes, and 24 hours. Lacritin enhanced metabolic activity was maximal at 1 minute (Fig. 2).

\section{BAK treatment of CRL-11515 cells increases autophagy}

One minute treatment with $0.004 \%$ BAK increased autophagic marker LC3-II more than treatment with $0.001 \%$ BAK (Fig. 3), consistent with decreased metabolic activity after BAK exposure (Fig. 1). 0.004\% BAK was accordingly chosen for subsequent autophagy experiments. Quantitation was performed by densitometry and expressed as the normalized ratio of LC3-II/GAPDH.

\section{Pre-treatment of CRL-11515 cells with lacritin rescues BAK induced autophagy}

Lacritin itself did not show any statistically significant positive or negative effect on autophagy in the absence of BAK-induced stress (Fig. 4).

Treating CRL-11515 cells with $1 \mathrm{nM}$ lacritin and $0.004 \%$ BAK simultaneously for one minute had no statistically significant effect, however pre-incubating CRL-11515 cells with $1 \mathrm{nM}$ lacritin for 24 hours prior to $0.004 \%$ BAK treatment for 1 minute significantly reduced LC3-II compared to 1 minute $0.004 \%$ BAK treatment alone (Fig. 5).

\section{Co- and pre-treatment of human corneal epithelial cells with lacritin increases cellular metabolic activity}

Subsequently, a series of experiments was performed combining $0.004 \%$ BAK and $1 \mathrm{nM}$ lacritin to determine if pre-treating with or co-administering lacritin would partially rescue BAK-suppressed cellular metabolic activity. Adding lacritin to BAK for 1 minute promoted a statistically significant increase in CRL-11515 cell metabolic activity compared to BAK alone. Pre-incubating the cells with lacritin for 24 hours was also effective (Fig. 6A). To 
confirm the cell line results, primary HCE cells were used and our data shows that lacritin at $1 \mathrm{nM}$ also had a protective effect against BAK-induced inhibition of metabolic activity in primary HCE cells (Fig. 6B).

\section{Discussion}

BAK is a preservative commonly used in multi-dose eye drops, such as those approved for the treatment of glaucoma. However, studies have shown ocular toxicity associated with BAK (1, 16-20). In the present study, the MTT assay was used to measure NADPHdependent cellular oxioreductase enzyme activity, which reflects mitochondrial activity and cell viability (21). Even at the lowest concentration tested, $0.001 \%$, there was significant loss of cellular metabolic activity, and we demonstrated that this decrease in cellular metabolic activity was present even at short exposure times. The concentration range of BAK in topical agents is typically between $0.004 \%$ and $0.025 \%$ (22), up to 25 times higher than the lowest concentration employed in this study. In addition, since the half-life of BAK is 20 hours in corneal epithelial tissues (23), this may cause prolonged damage to the ocular surface in patients chronically using eye drops. However, BAK is still used in ophthalmic drops because preservative free eye drops are at risk of contamination, which can lead to serious ocular infections $(24,25)$. Thus, it is important to find an agent that protects against BAK toxicity. The present study was designed to investigate whether lacritin is capable of protecting the ocular surface from the cytotoxic effects of BAK.

Lacritin has both prosecretory and mitogenic properties and it is selectively downregulated in dry eye. However, the effect of lacritin on BAK-induced cytotoxicity has not been established. In the present study, we demonstrated lacritin alone increases cellular metabolic activity of HCE cells in a biphasic manner. Concomitant and pre-treatment with lacritin and BAK significantly increased cellular metabolic activity compared to HCE cells treated with BAK alone. Our results suggest that lacritin increases cellular viability and protects HCE cells from BAK-induced cellular toxicity.

Autophagy is a cellular process in which damaged or dysfunctional organelles and protein aggregates are removed by the formation of autophagosomes, which requires the participation of autophagy-related proteins and microtubule-associated protein light chain 3II (LC3-II) $(14,15)$. The level of autophagy is tightly controlled to achieve cellular homeostasis. However, massive induction of autophagy may promote cell death and injury. It has been shown that BAK induces excessive autophagy of conjunctival epithelial cells (26). Notably, lacritin increases LC3-II degradation in human corneal epithelial cells stressed with interferon- $\gamma$ and TNF- $\alpha$ (10). To our knowledge, it is not known whether BAK induces autophagy in human corneal epithelial cells. Our data showed that BAK increases LC3-II expression, indicating activation of autophagy. Interestingly, lacritin alone does not significantly alter LC3-II expression in HCE cells. Notably, pre-treatment with lacritin for 24 hours decreased LC3-II expression induced by BAK. Our data suggests that lacritin inhibits autophagy induced by BAK in HCE cells.

We chose to use an immortalized cell line in our study to avoid inconsistency that occurs in primary corneal epithelial cultures due to replicative genetic changes (27). We employed the 
CRL-11515 immortalized cell line, which has the advantage of displaying the same cytotoxic response as primary cultured HCE cells to eight $\beta$-blockers including propranolol, alprenolol, and metoprolol (28). However, parallel experiments conducted using primary HCE cells showed the same results, indicating that the protective effects of lacritin are not an anomaly of the CRL-11515 immortalised cell line,

In summary, this study has shown that lacritin offers protection to cultured HCE cells stressed with BAK. Lacritin increased cell viability and reduced autophagy during BAK administration. BAK is a common preservative found in eye drops, and has been shown to cause ocular surface toxicity in patients frequently using multi-dose eye drops. Our study suggests lacritin has the potential to be a natural topical adjunctive therapy and possibly increase compliance in patients using eye drops that contain BAK.

\section{References}

1. Dutot M, Pouzaud F, Larosche I, Brignole-Baudouin F, Warnet JM, Rat P. Fluoroquinolone eye drop-induced cytotoxicity: role of preservative in $\mathrm{P} 2 \mathrm{X} 7$ cell death receptor activation and apoptosis. Invest Ophthalmol Vis Sci. 2006; 47(7):2812-9. [PubMed: 16799018]

2. Epstein SP, Chen D, Asbell PA. Evaluation of biomarkers of inflammation in response to benzalkonium chloride on corneal and conjunctival epithelial cells. J Ocul Pharmacol Ther. 2009; 25(5):415-24. [PubMed: 19857103]

3. De Saint Jean M, Brignole F, Bringuier AF, Bauchet A, Feldmann G, Baudouin C. Effects of benzalkonium chloride on growth and survival of Chang conjunctival cells. Invest Ophthalmol Vis Sci. 1999; 40(3):619-30. [PubMed: 10067965]

4. Sosne G, Albeiruti AR, Hollis B, Siddiqi A, Ellenberg D, Kurpakus-Wheater M. Thymosin beta4 inhibits benzalkonium chloride-mediated apoptosis in corneal and conjunctival epithelial cells in vitro. Exp Eye Res. 2006; 83(3):502-7. [PubMed: 16630613]

5. Sanghi S, Kumar R, Lumsden A, Dickinson D, Klepeis V, Trinkaus-Randall V, et al. cDNA and genomic cloning of lacritin, a novel secretion enhancing factor from the human lacrimal gland. $\mathrm{J}$ Mol Biol. 2001; 310(1):127-39. [PubMed: 11419941]

6. Zhou L, Beuerman RW, Foo Y, Liu S, Ang LP, Tan DT. Characterisation of human tear proteins using high-resolution mass spectrometry. Ann Acad Med Singapore. 2006; 35(6):400-7. [PubMed: 16865190]

7. Wang, N.; Raab, RW.; McKown, RL.; Laurie, GW. Invest Ophthalmol Vis Sci. ARVO; Fort Lauderdale, FL: 2011. Lacritin rapidly suppresses inflammatory stress in human corneal epithelial cells.

8. Wang J, Wang N, Xie J, Walton SC, McKown RL, Raab RW, et al. Restricted epithelial proliferation by lacritin via PKCalpha-dependent NFAT and mTOR pathways. J Cell Biol. 2006; 174(5):689-700. [PubMed: 16923831]

9. Samudre S, Lattanzio FA Jr, Lossen V, Hosseini A, Sheppard JD Jr, McKown RL, et al. Lacritin, a novel human tear glycoprotein, promotes sustained basal tearing and is well tolerated. Invest Ophthalmol Vis Sci. 2011; 52(9):6265-70. [PubMed: 21087963]

10. Wang N, Zimmerman K, Raab RW, McKown RL, Hutnik CM, Talla V, et al. Lacritin rescues stressed epithelia via rapid forkhead box O3 (FOXO3)-associated autophagy that restores metabolism. J Biol Chem. 2013; 288(25):18146-61. [PubMed: 23640897]

11. Nichols JJ, Green-Church KB. Mass spectrometry-based proteomic analyses in contact lens-related dry eye. Cornea. 2009; 28(10):1109-17. [PubMed: 19770725]

12. Koo BS, Lee DY, Ha HS, Kim JC, Kim CW. Comparative analysis of the tear protein expression in blepharitis patients using two-dimensional electrophoresis. J Proteome Res. 2005; 4(3):719-24. [PubMed: 15952718]

13. McKown RL, Wang N, Raab RW, Karnati R, Zhang Y, Williams PB, et al. Lacritin and other new proteins of the lacrimal functional unit. Exp Eye Res. 2009; 88(5):848-58. [PubMed: 18840430] 
14. Levine B, Deretic V. Unveiling the roles of autophagy in innate and adaptive immunity. Nat Rev Immunol. 2007; 7(10):767-77. [PubMed: 17767194]

15. Galluzzi L, Vicencio JM, Kepp O, Tasdemir E, Maiuri MC, Kroemer G. To die or not to die: that is the autophagic question. Curr Mol Med. 2008; 8(2):78-91. [PubMed: 18336289]

16. Ishibashi T, Yokoi N, Kinoshita S. Comparison of the short-term effects on the human corneal surface of topical timolol maleate with and without benzalkonium chloride. J Glaucoma. 2003; 12(6):486-90. [PubMed: 14646684]

17. Chung SH, Lee SK, Cristol SM, Lee ES, Lee DW, Seo KY, et al. Impact of short-term exposure of commercial eyedrops preserved with benzalkonium chloride on precorneal mucin. Mol Vis. 2006; 12:415-21. [PubMed: 16688112]

18. Noecker RJ, Herrygers LA, Anwaruddin R. Corneal and conjunctival changes caused by commonly used glaucoma medications. Cornea. 2004; 23(5):490-6. [PubMed: 15220734]

19. Cha SH, Lee JS, Oum BS, Kim CD. Corneal epithelial cellular dysfunction from benzalkonium chloride (BAC) in vitro. Clin Experiment Ophthalmol. 2004; 32(2):180-4. [PubMed: 15068436]

20. Guo Y, Satpathy M, Wilson G, Srinivas SP. Benzalkonium chloride induces dephosphorylation of Myosin light chain in cultured corneal epithelial cells. Invest Ophthalmol Vis Sci. 2007; 48(5): 2001-8. [PubMed: 17460253]

21. van Meerloo J, Kaspers GJ, Cloos J. Cell sensitivity assays: the MTT assay. Methods Mol Biol. 2011; 731:237-45. [PubMed: 21516412]

22. Baudouin C, Labbe A, Liang H, Pauly A, Brignole-Baudouin F. Preservatives in eyedrops: the good, the bad and the ugly. Prog Retin Eye Res. 2010; 29(4):312-34. [PubMed: 20302969]

23. Champeau, EJEHF. The effect of ophthalmic preservatives on the ocular surface: conjunctival and corneal uptake and distribution of Benzalkonium chloride and chlorhexidine digluconate. JHF, editor. Dry eye Institute, Inc; 1986.

24. Rahman MQ, Tejwani D, Wilson JA, Butcher I, Ramaesh K. Microbial contamination of preservative free eye drops in multiple application containers. Br J Ophthalmol. 2006; 90(2):13941. [PubMed: 16424520]

25. Templeton WC 3rd, Eiferman RA, Snyder JW, Melo JC, Raff MJ. Serratia keratitis transmitted by contaminated eyedroppers. Am J Ophthalmol. 1982; 93(6):723-6. [PubMed: 7046448]

26. Buron N, Micheau O, Cathelin S, Lafontaine PO, Creuzot-Garcher C, Solary E. Differential mechanisms of conjunctival cell death induction by ultraviolet irradiation and benzalkonium chloride. Invest Ophthalmol Vis Sci. 2006; 47(10):4221-30. [PubMed: 17003409]

27. Kuilman T, Michaloglou C, Mooi WJ, Peeper DS. The essence of senescence. Genes Dev. 2010; 24(22):2463-79. [PubMed: 21078816]

28. Cheong HI, Johnson J, Cormier M, Hosseini K. In vitro cytotoxicity of eight beta-blockers in human corneal epithelial and retinal pigment epithelial cell lines: comparison with epidermal keratinocytes and dermal fibroblasts. Toxicol In Vitro. 2008; 22(4):1070-6. [PubMed: 18316173]

Curr Eye Res. Author manuscript; available in PMC 2015 June 01. 


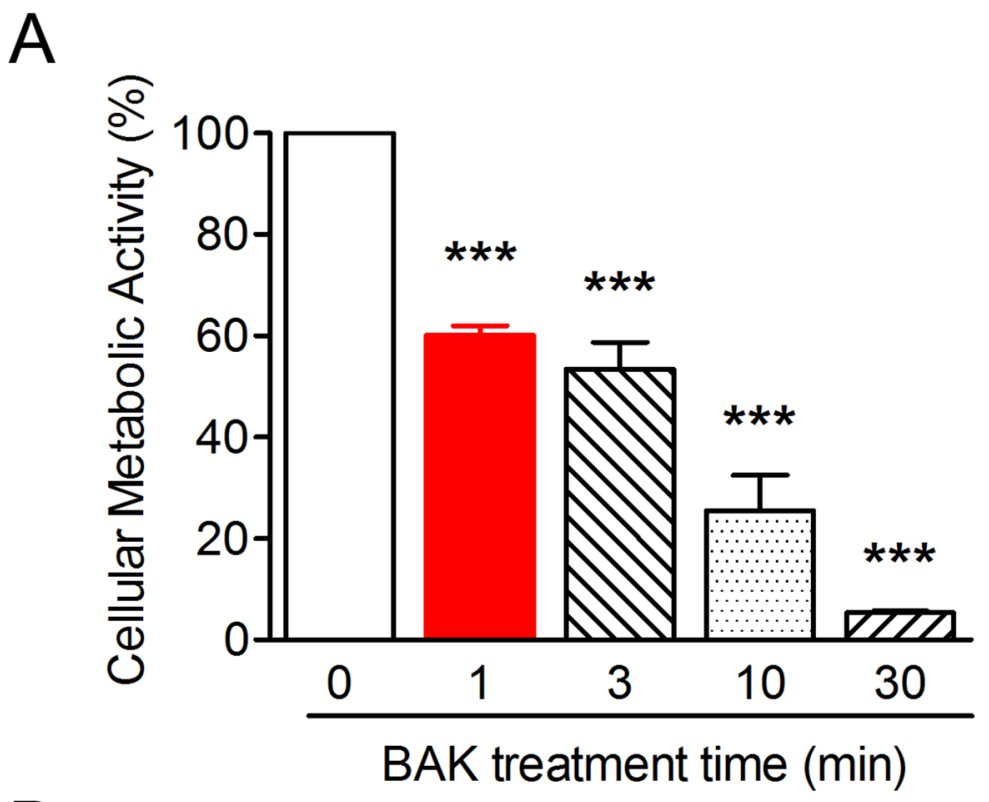

B

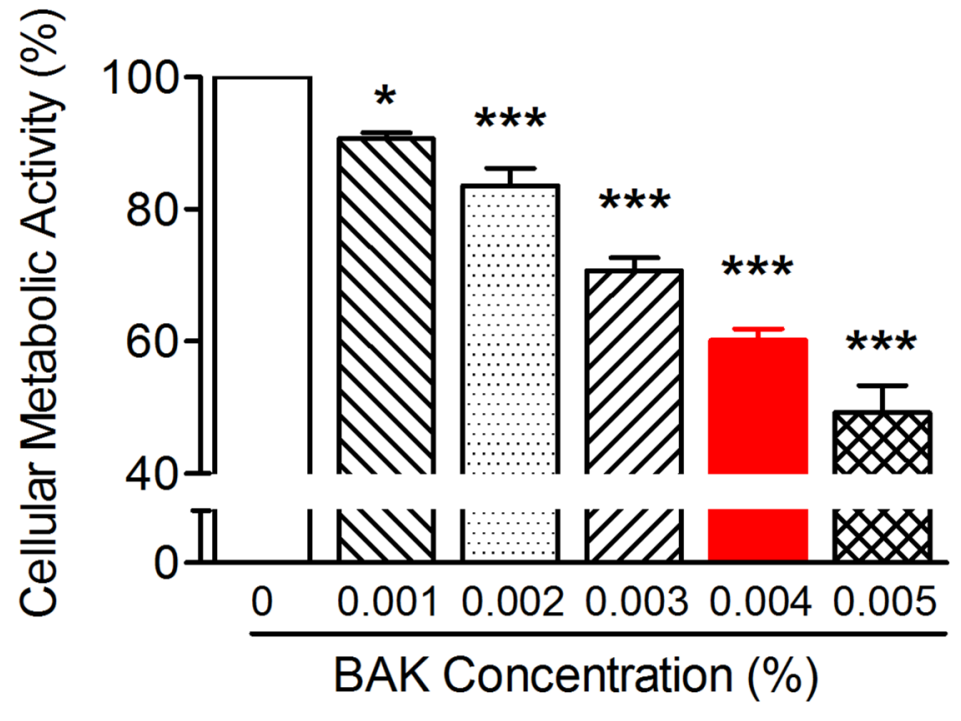

Figure 1.

BAK-induced suppression of CRL-11515 cellular metabolic activity is time and dose dependent. (Fig. 1A) CRL-11515 cells were treated with 0.004\% BAK for 0, 1, 3, 10 and 30 minutes and cellular metabolic activity was assessed by the MTT assay ( $* * * P<0.001$ vs. 0 minute; $n=4)$. (Fig. 1B) Cells were treated with increasing concentrations of BAK for 1 minute and cellular metabolic activity was assessed by the MTT assay $(* P<0.05, * * * P<$ 0.001 vs. $0 \% ; n=4)$. 


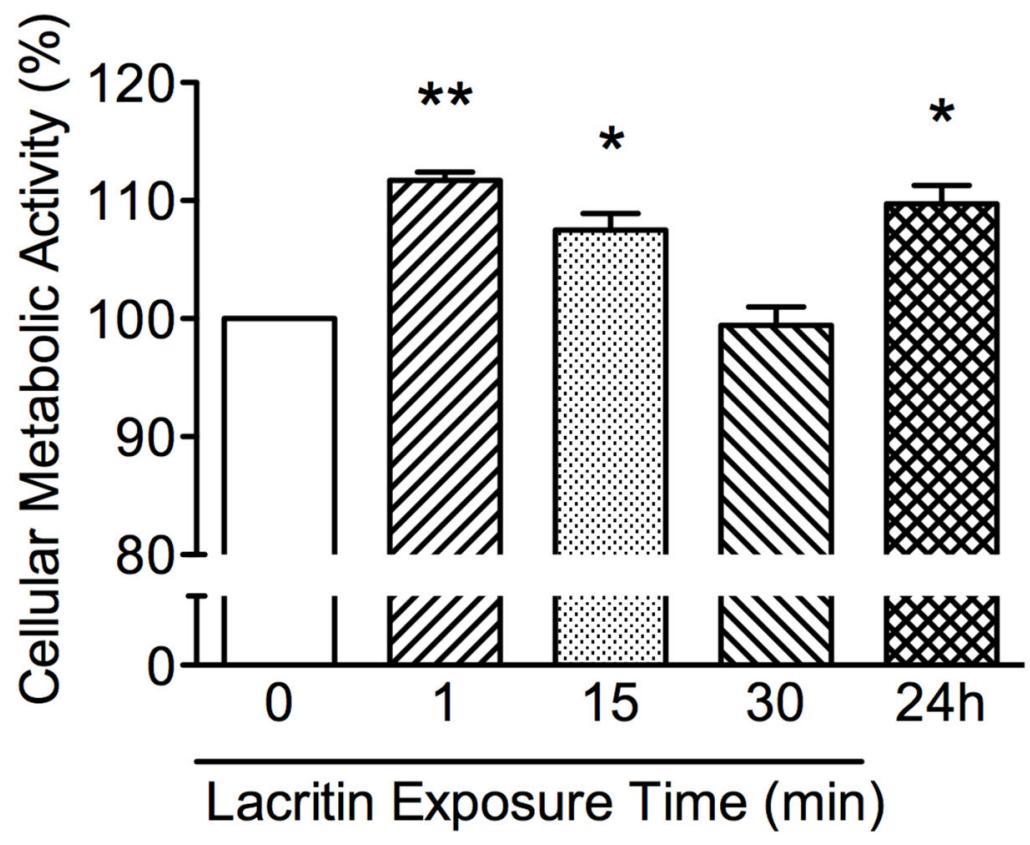

Figure 2.

Lacritin enhances metabolic activity of CRL-11515 cells in a time dependent manner. Cellular metabolic activity following 1 minute, 15 minutes 30 minutes, and 24 hour exposure to $1 \mathrm{nM}$ lacritin shows a biphasic curve, as measured by MTT assay. Data are expressed as a percentage of control. (**P<0.001, $* P<0.05 ; n=4)$. 


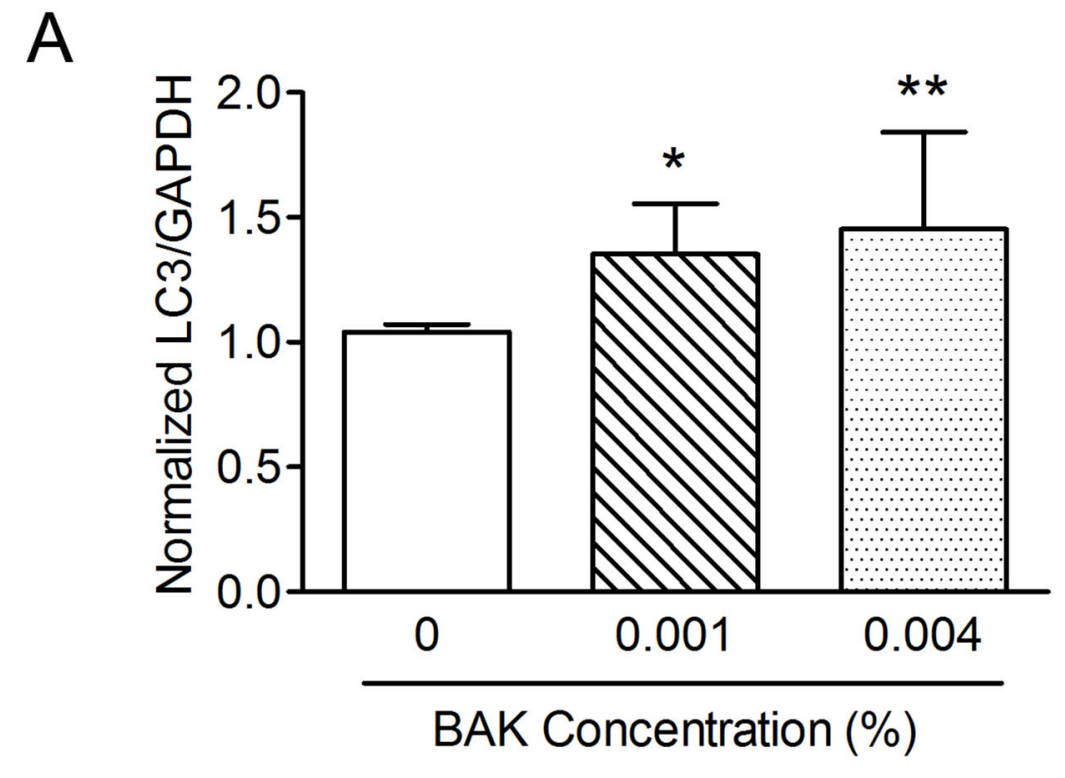

B
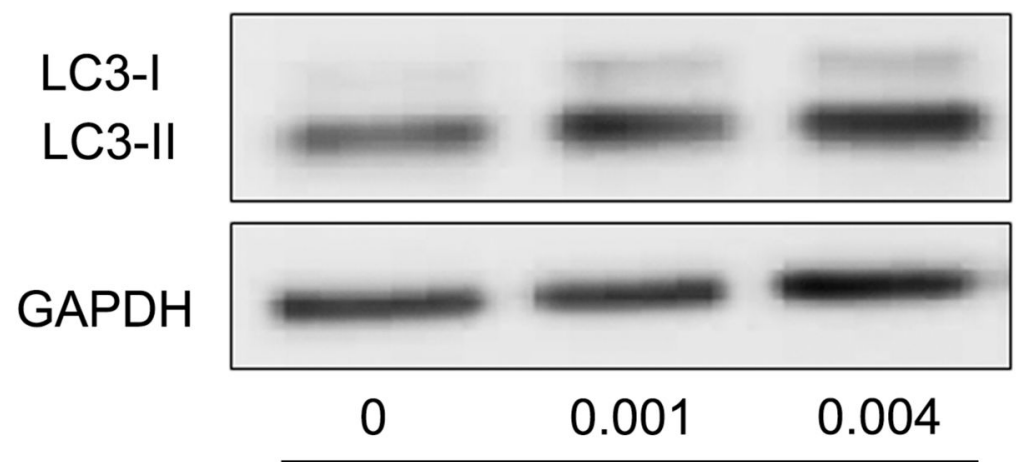

BAK Concentration (\%)

Figure 3.

BAK treatment of CRL-11515 cells increases autophagy, as suggested by increased LC3-II. (Fig. 3A) CRL-11515 cells treated with BAK $(0.001 \%, 0.004 \%)$ for 1 minute increased cellular lipidated LC3, known as 'LC3-II' with increasing concentration of BAK $(* * P<$ $0.01,{ }^{*} P<0.05 ; n=6$ ). (Fig. 3B) Western blot of LC3-II with GAPDH as loading control. 
A

B

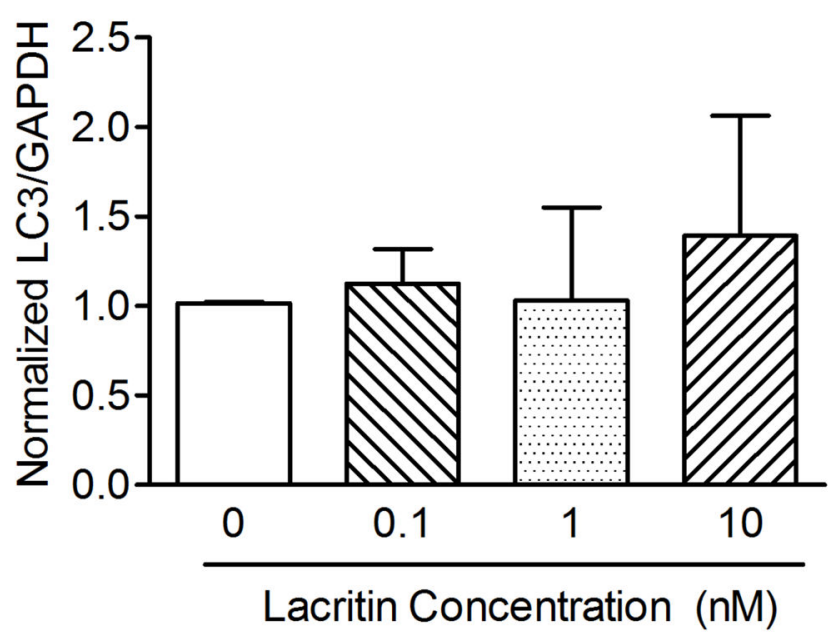

\section{LC3-I \\ LC3-II}

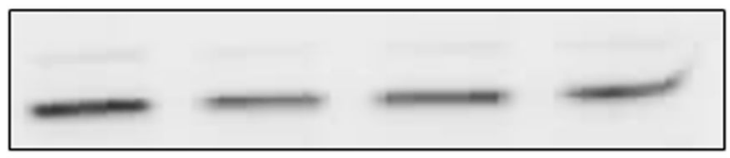

GAPDH



Lacritin Concentration (nM)

Figure 4.

Lacritin treatment alone does not affect levels of cellular LC3-II. (Fig. 4A) LC3-II detection in CRL-11515 cells treated with lacritin (0.1-10 nM) for 24 hours suggests no significant change in autophagy $(n=5)$. (Fig. 4B) Western blot of LC3-II with GAPDH as control. 

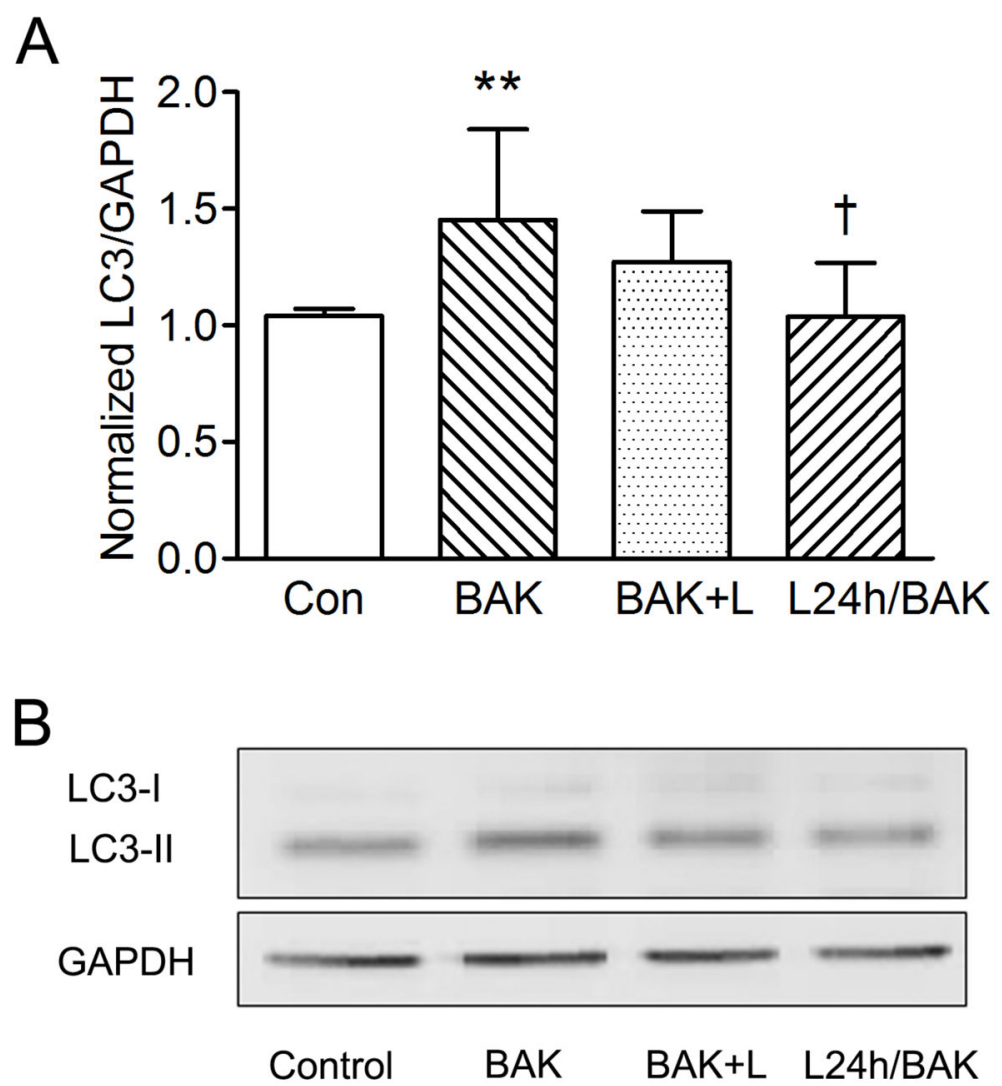

Figure 5.

Lacritin treatment of CRL-11515 cells rescues BAK induced autophagy. (Fig 5A) Apparent level of LC3-II increased in CRL-11515 cells treated with BAK $(0.004 \%)$ compared to control ( $* * P<0.01$ vs. control; $n=5$ ). L24h/BAK significantly reduced LC3-II compared to BAK treated cells $(\dagger P<0.05$ vs. BAK; $n=5$ ). (Fig. 5B) Western Blot of LC3-II with GAPDH as loading control. $\mathrm{BAK}=\mathrm{BAK}$ for $1 \mathrm{~min}$. $\mathrm{BAK}+\mathrm{L}=\mathrm{BAK}$ and lacritin for $1 \mathrm{~min}$. L24h/BAK = Pre-incubation with lacritin for 24 hours, followed by BAK treatment for 1 $\min$. 


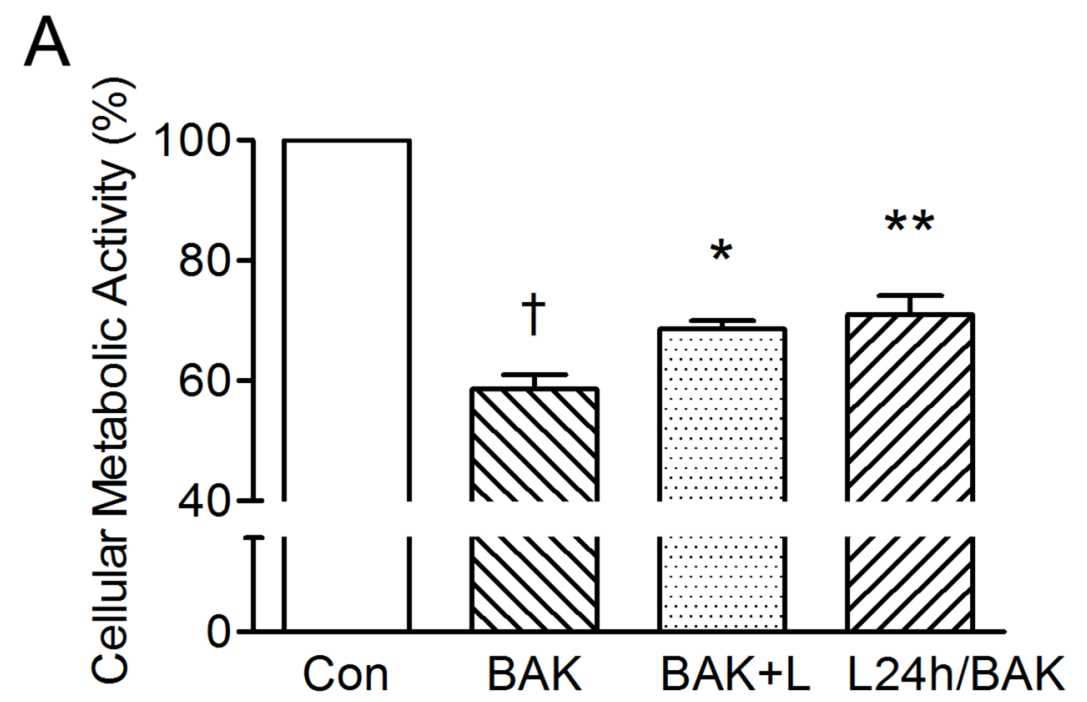

B

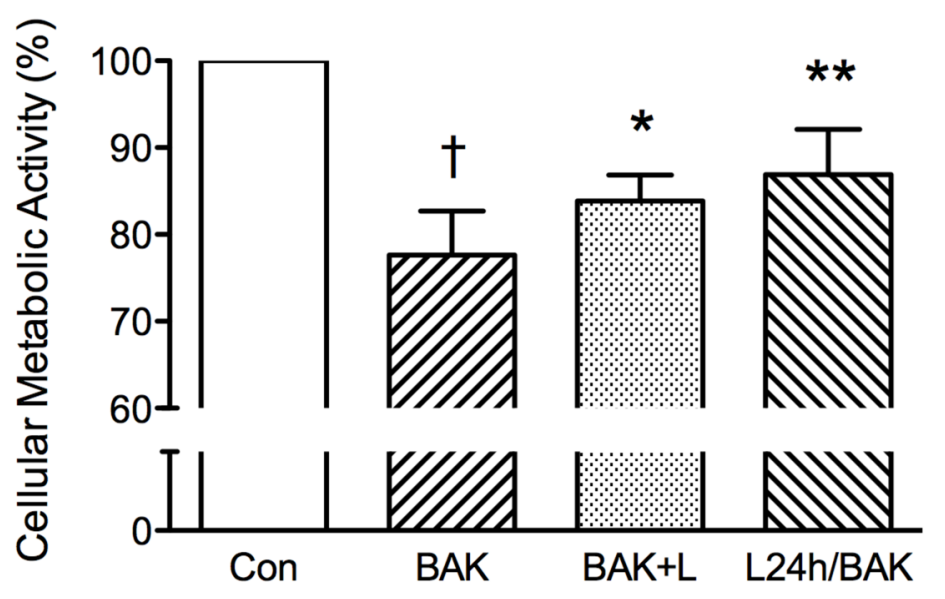

Figure 6.

Co- and pre-treatment human corneal epithelial cells with lacritin increases cellular metabolic activity. Treating (Fig. 6A) CRL-11515 cells and (Fig. 6B) primary HCE cells with $0.004 \%$ BAK significantly reduced cellular metabolic activity $(\dagger P<0.001$ vs. control; $n=5)$. Simultaneous administration of $0.004 \%$ BAK and $1 \mathrm{nM}$ lacritin significantly increased cellular metabolic activity by $10 \%(* P<0.05$ vs. BAK; $n=5)$. Pre-treatment of HCE cells with $1 \mathrm{nM}$ lacritin increased cellular metabolic activity by $12 \%$ compared to BAK treatment alone ( $* * P<0.01$ vs. BAK; $n=5$ ). BAK $=\mathrm{BAK}$ for 1 min. $\mathrm{BAK}+\mathrm{L}=\mathrm{BAK}$ and lacritin for $1 \mathrm{~min}$. L24h/BAK = Pre-incubation with lacritin for 24 hours, followed by BAK treatment for $1 \mathrm{~min}$. 